\title{
Risk factor analysis of acute kidney injury after one-stop hybrid coronary revascularization
}

\author{
Dongjie Li ${ }^{1 \#}, \mathrm{Jie}_{\mathrm{Gao}}{ }^{1 \#}$, Yulin Guo ${ }^{1}$, Yanxiong Jia ${ }^{1}$, Xiangguang An ${ }^{1}$, Yan Liu ${ }^{1}$, Lefeng Wang ${ }^{2}$, Pixiong Su ${ }^{1}$ \\ ${ }^{1}$ Department of Cardiac Surgery, Heart Center, Beijing Chaoyang Hospital, Capital Medical University, Beijing, China; ${ }^{2}$ Heart Center and Beijing \\ Key Laboratory of Hypertension, Beijing Chaoyang Hospital, Capital Medical University, Beijing, China \\ Contributions: (I) Conception and design: D Li, P Su; (II) Administrative support: L Wang, Y Liu, P Su; (III) Provision of study materials or patients: \\ D Li, J Gao, Y Guo, X An; (IV) Collection and assembly of data: D Li, Y Jia; (V) Data analysis and interpretation: D Li, J Gao; (VI) Manuscript \\ writing: All authors; (VII) Final approval of manuscript: All authors. \\ \#These authors contributed equally to this work. \\ Correspondence to: Lefeng Wang; Pixiong Su. No. 8 Gong Ti South Road, Chaoyang District, Beijing 100020, China. Email: wlf311@126.com; \\ supixiong1130@163.com.
}

Backgrounda One-stop hybrid coronary revascularization (HCR) combines coronary artery bypass grafting (CABG) and percutaneous coronary intervention (PCI) procedures simultaneously. Acute kidney injury (AKI) is a common complication after CABG or PCI. This study explored the risk factors for developing AKI after one-stop HCR.

Methods: A total of 123 consecutive patients who underwent one-stop HCR between April 2018 and November 2020 were included in this single-center retrospective study. Postoperative AKI was observed in 11 patients (the AKI group), and 112 patients did not develop postoperative AKI (the non-AKI group). Baseline characteristics and perioperative variables were extracted from the electronic medical records and statistically analyzed.

Results: Postoperative AKI occurred in 11 (8.9\%) patients. Compared to patients who did not develop

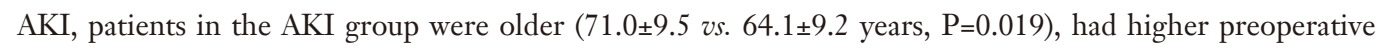
creatinine levels $(92.6 \pm 16.8$ vs. $69.8 \pm 14.4 \mathrm{mmol} / \mathrm{L}, \mathrm{P}<0.001)$, experienced a greater volume of postoperative drainage on the first day $\{850 \mathrm{~mL}[410,1,500]$ vs. $500 \mathrm{~mL}[321,700], \mathrm{P}=0.045\}$, had a higher proportion of chronic renal insufficiency (eGFR $\left.<60 \mathrm{~mL} / \mathrm{min} / 1.73 \mathrm{~m}^{2}\right)(36.4 \%$ vs. $7.1 \%, \mathrm{P}=0.012)$, and had more perioperative transfusions (63.6\% vs. $22.3 \%, \mathrm{P}=0.007)$. Multivariate logistics analyses revealed that advanced age [odds ratio (OR) 5.44, $\mathrm{P}=0.014$ ] and perioperative transfusions (OR 4.62, $\mathrm{P}=0.028$ ) were independent risk factors for developing AKI after one-stop HCR.

Conclusions: One-stop HCR did not increase the incidence of postoperative AKI in our center. Advanced age ( $\geq 75$ years) and perioperative transfusions were independent risk factors for developing AKI after onestop HCR. Further studies need to be conducted to confirm the risk factors of AKI after HCR procedures.

Keywords: Coronary artery bypass grafting (CABG); percutaneous coronary intervention (PCI); hybrid coronary revascularization (HCR); acute kidney injury (AKI); risk factor

Submitted Apr 20, 2021. Accepted for publication Jun 23, 2021.

doi: $10.21037 /$ apm-21-959

View this article at: https://dx.doi.org/10.21037/apm-21-959 


\section{Introduction}

Coronary artery bypass grafting (CABG) and percutaneous coronary intervention (PCI) are classic treatment strategies for patients with multivessel coronary artery disease (1). Hybrid coronary revascularization (HCR) was first developed in 1996 and combined CABG with PCI, thereby benefiting from the cumulative advantages while avoiding the relative deficiencies of CABG and PCI (2). HCR can be performed at a hybrid suite in a single operative setting (one-stop or simultaneous HCR) or performed within hours, days, or even weeks apart for the two procedures by using conventional operating theaters and catheterization laboratories (staged HCR) (3). Acute kidney injury (AKI) is a common and significant complication after CABG and PCI, and is associated with increased postoperative morbidity and mortality $(4,5)$.

Since one-stop HCR involves CABG and PCI being performed simultaneously, this may theoretically increase the incidence of postoperative AKI. However, there have been no studies examining the clinical characteristics and risk factors associated with postoperative AKI in onestop HCR. Therefore, this study aims to identify the perioperative risk factors of developing AKI after isolated one-stop HCR. We present the following article in accordance with the STROBE reporting checklist (available at https://dx.doi.org/10.21037/apm-21-959).

\section{Methods}

\section{Study population}

This single-center retrospective study was conducted at Beijing Chaoyang Hospital, Capital Medical University, China. A total of 125 consecutive patients who underwent one-stop HCR at our institution between April 2018 and November 2020 were retrospectively included in this analysis. Selection criterias of patients underwent one-stop HCR were: patients with multivessel disease in whom the left anterior descending artery (LAD) lesion was not suitable for PCI but was suitable for surgical revascularization and in whom the non-LAD lesions were amenable to PCI; patients who were not suitable candidates for traditional CABG, such as patients with poor right coronary or circumflex arteries for bypass, contraindications for sternotomy, porcelain aorta, a lack of acceptable conduits, and a desire for minimally invasive procedure. Patients with end-stage renal disease (ESRD) or patients who were receiving renal replacement therapy (RRT) before the operation were excluded. Finally, 123 patients were enrolled in the study, of which 11 patients (8.9\%) developed postoperative AKI (AKI group), and 112 patients did not develop postoperative AKI (non-AKI group). The study was conducted in accordance with the Declaration of Helsinki (as revised in 2013). The study was approved by local research ethics board of Chaoyang Hospital (No.: 2021-D-5) and individual consent for this retrospective analysis was waived.

\section{Data definitions}

Perioperative baseline variables are shown in Table 1. Demographic parameters and surgical characteristics including age, gender, body mass index (BMI), preoperative creatinine, estimated glomerular filtration rate (eGFR), smoking history, hypertension, hyperlipidemia, diabetes mellitus, insulin-dependent diabetes, chronic obstructive pulmonary disease (COPD), peripheral vascular disease, previous stroke, previous myocardial infarction (MI), previous PCI, recent acute myocardial infarction (AMI), left main disease, left ventricular ejection fraction (LVEF), left ventricular end-diastolic dimension (LVDD), preoperative intra-aortic balloon pump (IABP), dose of contrast medium, perioperative transfusions, postoperative drainage on the first day, and reoperation for bleeding, were retrospectively collated from the electronic medical records of Chaoyang Hospital. Key data elements were defined according to the Society of Thoracic Surgeons database and the 2013 "ACCF/AHA Key Data Elements and Definitions for Measuring the Clinical Management and Outcomes of Patients with Acute Coronary Syndromes and Coronary Artery Disease" (6).

The definition and classification of postoperative AKI were following the 2012 Kidney Disease Improving Global Outcomes (KDIGO) guideline. The AKI definition satisfies one of the following: an increase in serum creatinine by $\geq 0.3 \mathrm{mg} / \mathrm{dL}$ ( $\geq 26.5 \mathrm{mmol} / \mathrm{L}$ ) within 48 hours; an increase in serum creatinine to $\geq 1.5$ times baseline within the previous 7 days; and urine volume $\leq 0.5 \mathrm{~mL} / \mathrm{kg} / \mathrm{h}$ for 6 hours (7).

\section{Interventions}

For the HCR procedures, minimally invasive direct coronary artery bypass (MIDCAB) and PCI were simultaneously performed in hybrid operation room. Surgical procedures were all performed by one experienced surgeon (Pixiong Su). Left internal mammary artery (LIMA) was harvested via direct vision or assisted by endoscopy 
Table 1 Baseline characteristics of patients in AKI group and non-AKI group

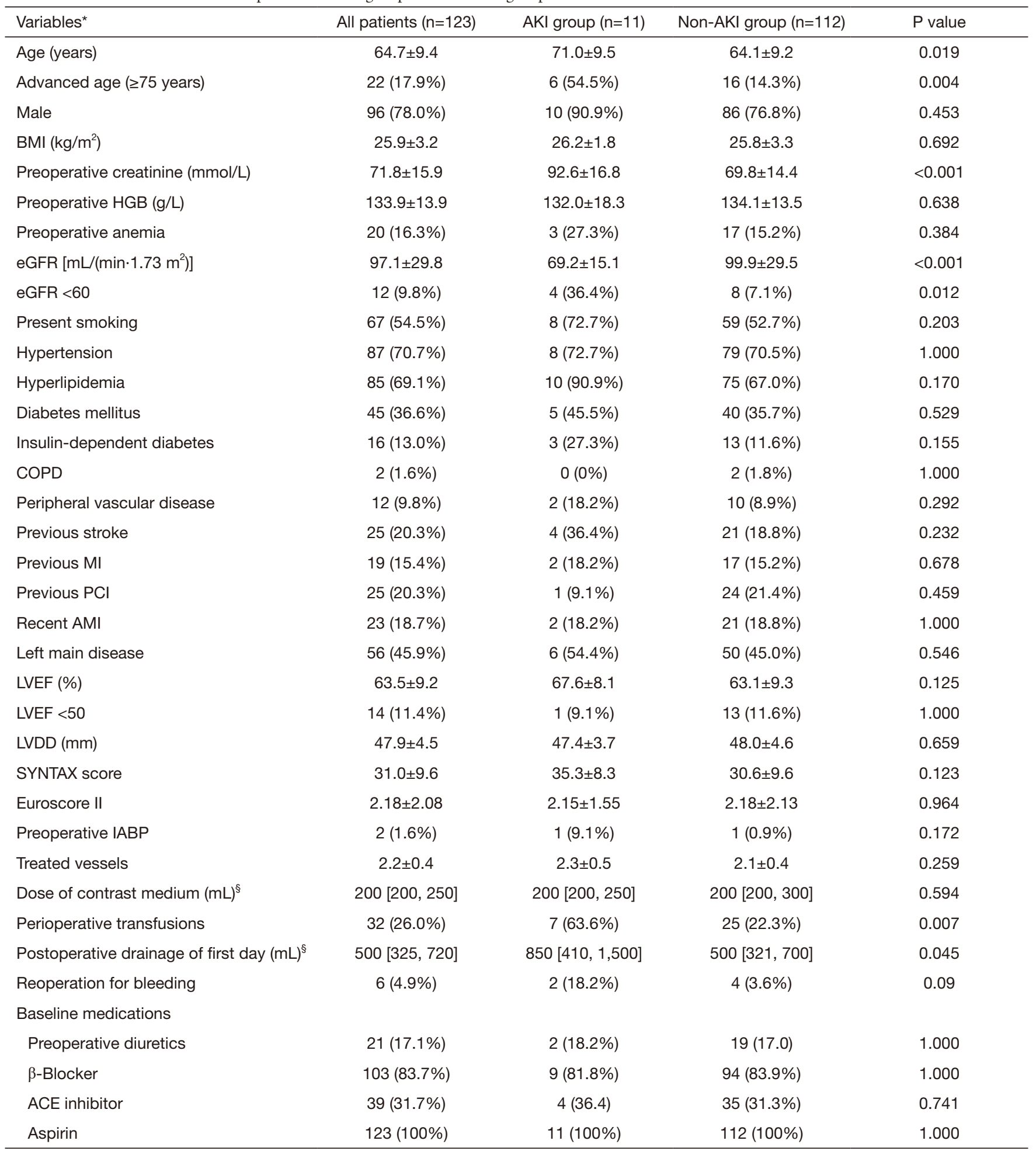

*, continuous variables with normal distribution are presented as mean \pm SD. ${ }^{\S}$, presented as median $\left[\mathrm{P}_{25}, \mathrm{P}_{75}\right]$. AKI, acute kidney injury; BMI, body mass index; HGB, hemoglobin; eGFR, estimated glomerular filtration rate; COPD, chronic obstructive pulmonary disease; MI, myocardial infarction; AMI, acute myocardial infarction; PCI, percutaneous coronary intervention; LVEF, left ventricular ejection fraction; LVDD, left ventricular end-diastolic dimension; IABP, intra-aortic balloon pump. 
through a 5 to $7 \mathrm{~cm}$ anterolateral minithoracotomy in the fourth or fifth intercostal space. The LIMA to left anterior descending artery (LAD) anastomosis was then performed through the same incision with the same technique as conventional off-pump CABG via sternotomy. The PCI procedures were performed using standard techniques. LIMA-LAD graft patency was immediately confirmed by angiography after the chest closure.

Drug-eluting stents (DES) or drug-coated balloons (DCB) were implanted in the non-LAD lesions. Iodixanol was used as the contrast medium in the PCI procedures.

\section{Statistical analysis}

Continuous variables are expressed as mean and standard deviation, and categorical variables are expressed as a proportion. The $t$-test or Mann-Whitney $\mathrm{U}$ test was used to compare the variables between the AKI and non-AKI groups for continuous variables, and the chi-squared test or Fisher exact test was used for categorical variables. The risk factors associated with postoperative AKI were evaluated via univariate logistic regression, and multiple logistic regression analyses identified the independent risk factors for AKI. All statistical data analyses were conducted using the SPSS software package (IBM SPSS Statistics for Windows, Version 22.0), and a $\mathrm{P}$ value $<0.05$ was considered statistically significant.

\section{Results}

\section{Baseline characteristics}

A total of 123 patients who underwent HCR were included in the study, and no patients required conversion to sternotomy. The mean age was $64.7 \pm 9.4$ years, and 96 (78.0\%) patients were male. The mean preoperative serum creatinine was $71.8 \pm 15.9 \mathrm{mmol} / \mathrm{L}$, the mean eGFR level was $97.1 \pm 29.8 \mathrm{~mL} / \mathrm{min} / 1.73 \mathrm{~m}^{2}$, the mean number of treated vessels was $2.2 \pm 0.4$, and the median volume of postoperative drainage on the first day was $500 \mathrm{~mL}, 12(9.8 \%)$ patients had eGFR levels less than $60 \mathrm{~mL} / \mathrm{min} / 1.73 \mathrm{~m}^{2}$ and 32 (26.0\%) patients received perioperative transfusions. Baseline characteristics and postoperative outcome data of the patients are presented in Table 1 .

Postoperative AKI occurred in 11 (8.9\%) patients according to the KDIGO guideline, of which 8 (72.7\%), 1 (9.1\%), and $2(18.2 \%)$ patients developed stage 1, 2, and 3 AKI, respectively. Two patients required continuous renal replacement therapy (CRRT) and both patients died during the hospital stay. The overall mortality was $1.6 \%$ in all patients and $18.1 \%$ in the AKI group. All other AKI patients recovered completely at discharge. Comparing patients in the AKI group and the non-AKI group, there were no differences in gender, BMI, preoperative hemoglobin, preoperative anemia, LVEF, heart failure, SYNTAX score, Euroscore II, dose of contrast medium, and baseline

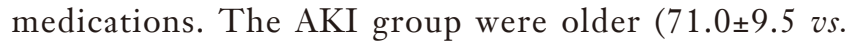
64.1 \pm 9.2 years, $\mathrm{P}=0.019)$, had higher preoperative creatinine levels $(92.6 \pm 16.8$ vs. $69.8 \pm 14.4 \mathrm{mmol} / \mathrm{L}, \mathrm{P}<0.001)$, had a greater volume of postoperative drainage on the first day $\{850 \mathrm{~mL}[410,1,500]$ vs. $500 \mathrm{~mL}[321,700], \mathrm{P}=0.045\}$, had a higher proportion of chronic renal insufficiency defined as eGFR $<60 \mathrm{~mL} / \mathrm{min} / 1.73 \mathrm{~m}^{2}(36.4 \%$ vs. $7.1 \%, \mathrm{P}=0.012)$, and higher rates of perioperative transfusions $(63.6 \%$ vs. $22.3 \%$, $\mathrm{P}=0.007)$ (Table 1).

\section{Logistic regression analysis for risk factors of AKI after HCR}

All the potential risk factors were involved in the univariate logistic regression analyses, variables that were statistically different in the univariate logistic regression analyses and baseline characteristic analysis were showed in Table 2. Advanced age $(\geq 75$ years $)(P=0.003)$, eGFR $<60 \mathrm{~mL} / \mathrm{min} / 1.73 \mathrm{~m}^{2}(\mathrm{P}=0.006)$, and perioperative transfusions $(\mathrm{P}=0.007)$ were risk factors for developing AKI. The volume of postoperative drainage on the first day was not a statistically significant risk factor. Then, logistic regression was performed with variables that had been significant in previous univariate analysis (advanced age, eGFR $<60$ and perioperative transfusions) for multivariate risk factors evaluation. Finally, it revealed that advanced age [odds ratio (OR) 5.44, $\mathrm{P}=0.014$ ] and perioperative transfusions ( $\mathrm{OR} 4.62, \mathrm{P}=0.028)$ were independent risk factors of developing AKI after one-stop HCR (Table 2).

\section{Discussion}

AKI is a well-known complication after cardiac surgery. The incidence of postoperative AKI ranges from $8.9 \%$ to $48.5 \%$ in patients undergoing isolated CABG (8-12), and $7.1 \%$ to $21.5 \%$ in patients who undergo PCI procedures (13-15). Postoperative AKI is associated with significantly increased risks of in-hospital MI, mortality, and other severe perioperative complications. It is also associated with significant medical expenses and risks of long-term major 
Table 2 Univariate and multivariate logistic regression of patients in AKI group and non-AKI group

\begin{tabular}{|c|c|c|c|c|c|}
\hline Variables & AKI group $(n=11)$ & $\begin{array}{l}\text { Non-AKI group } \\
(n=112)\end{array}$ & $\begin{array}{l}\text { Univariate } \\
\mathrm{P} \text { value }\end{array}$ & \multicolumn{2}{|c|}{ Multivariate } \\
\hline Advanced age ( $\geq 75$ years) & $6(54.5 \%)$ & $16(14.3 \%)$ & 0.003 & $5.44(1.40-21.14)$ & 0.014 \\
\hline eGFR $<60$ & $4(36.4 \%)$ & $8(7.1 \%)$ & 0.006 & - & 0.567 \\
\hline Perioperative transfusions & $7(63.6 \%)$ & $25(22.3 \%)$ & 0.007 & $4.62(1.18-18.10)$ & 0.028 \\
\hline
\end{tabular}

$\S$, presented as median $\left[\mathrm{P}_{25}, \mathrm{P}_{75}\right]$. AKI, acute kidney injury; OR, odds ratio; $\mathrm{Cl}$, confidence interval; eGFR, estimated glomerular filtration rate.

adverse events, including chronic kidney disease (CKD), end-stage renal disease, and death (15-17). In the present study, in-hospital mortality was $1.6 \%$ in all HCR patients and $18.1 \%$ in the AKI group, highlighting the severe adverse results of AKI.

It remains a complex mechanism for the pathophysiology of AKI following cardiac surgery and PCI, several major injury mechanisms have been proposed as the etiology in the development of AKI, including the direct nephrotoxic effects, hypoperfusion, ischaemia-reperfusion injury, increased viscosity of fluid in vascular and tubular cells, neurohumoral activation, oxidative stress, apoptosis, immune-inflammation injury, and epigenetic regulation $(18,19)$.

Mehta et al. suggested that the time interval between cardiac catheterization and elective $\mathrm{CABG}$ in postprocedural AKI was a major risk factor. This latter study demonstrated that patients in whom CABG was performed less than 1 day after cardiac catheterization had the highest risk of AKI (20). Wintgen et al. demonstrated that delayed postoperative re-angiography was an independent protective factor for the occurrence of post-angiography AKI in patients undergoing CABG (21). The one-stop HCR procedure combines surgical and catheterized revascularization, with the PCI procedure being performed immediately after the MIDCAB. Consequently, it can be hypothesized that HCR would increase the incidence of postoperative AKI compared with either CABG or PCI alone.

Few publications have demonstrated the incidence of AKI in one-stop HCR as it is only performed in a limited number of centers. Zhou and colleagues reported that patients undergoing the one-stop hybrid procedure had a higher incidence of AKI than patients undergoing off-pump CABG; however, this did not reach statistical significance (25.2\% vs. $17.6 \%, \mathrm{P}=0.13$ ) (22). Zhao et al. observed that the rate of AKI (defined as a $25 \%$ increase in creatinine at $72 \mathrm{~h}$ ) was $33 \%$ in one-stop HCR procedure (23). However, in the present study, $8.9 \%$ of patients developed AKI after one-stop HCR, which is slightly lower than the reported incidences of $\mathrm{AKI}$ in HCR, PCI, or CABG procedures and is contrary to our initial hypothesis. This outcome may be due to the following protective factors: (I) the MIDCAB procedure avoided cardiopulmonary bypass (CPB), decreased the systemic inflammatory response and ischemiareperfusion injury, and reduced the occurrence of AKI (24); (II) hemodynamic stability was maintained in all our patients resulting in adequate organ perfusion, and urine volume during the whole operation (25); (III) the contrast medium of choice in the PCI procedures was iso-osmolar nonionic iodixanol which has the same physiological osmolality as blood and has been shown to have the lowest risk for contrast-induced AKI, especially in patients with CKD and diabetes (5); (IV) the use of nephrotoxic drugs were avoided where possible due to the potential risk of kidney damage in one-stop HCR procedures.

Numerous studies have analyzed AKI in the setting of either CABG or PCI. Common risk factors for the development of AKI after CABG and PCI include advanced age, female sex, pre-existing CKD, COPD, diabetes mellitus, hypertension, hyperlipidemia, congestive heart failure, obesity, and the use of CPB and contrast medium $(15,26-28)$. However, to our knowledge, this current report is the first to discuss the risk factors involved in one-stop HCR procedures.

The present study identified that advanced age ( $\geq 75$ years) was an independent risk factor of developing AKI after one-stop HCR, and the risk of postoperative AKI increases significantly in elderly patients (OR 5.44, $\mathrm{P}=0.014)$. It is well-accepted that renal function declines with age $(27,29,30)$. Consistent with the literature, the mean eGFR in 
our study cohort was $68.8 \pm 17.7 \mathrm{~mL} / \mathrm{min} / 1.73 \mathrm{~m}^{2}$ in patients older than 75 years, and $103.2 \pm 28.4 \mathrm{~mL} / \mathrm{min} / 1.73 \mathrm{~m}^{2}$ in patients under 75 years old. Patients with low eGFR can withstand less ischemic damage before injury occurs. In addition, elderly patients have more comorbidities, which may also accelerate the progression of kidney injury.

Perioperative transfusions have been associated with postoperative AKI in cardiac surgery and PCI (31-33). The current study demonstrated that perioperative transfusions also increased the incidence of AKI in HCR procedures (OR 4.62, $\mathrm{P}=0.028$ ). The rate of AKI was $21.9 \%$ in patients receiving transfusions and only $4.4 \%$ in patients without transfusions. Most of the transfusions were required because of bleeding. The mean postoperative drainage on the first day was $1,038 \pm 621 \mathrm{~mL}$ in transfused patients and $499 \pm 408 \mathrm{~mL}$ in patients without transfusions. Appropriate antiplatelet therapy to balance the risk of stent thrombosis and surgical bleeding in one-stop HCR is challenging (34). Thus, one-stop HCR has different anticoagulant strategies. Aspirin $(100 \mathrm{mg} /$ day) was not discontinued preoperatively. Protamine was administrated at the end of MIDCAB, then a loading dose of clopidogrel was administered via a nasogastric tube before the closure of the thorax. An activated clotting time greater than 250 seconds was obtained by a second administration of unfractionated heparin in the PCI procedure. After surgery, all patients were treated with $100 \mathrm{mg}$ aspirin within 6 hours if there were no contraindications, and dual antiplatelet therapy was administered. These anticoagulation strategies may increase the risk of bleeding compared with isolated CABG or PCI, which may challenge the hemostasis techniques for the cardiac surgeon and lead to bleeding events.

The composition and volume of blood transfusions were based on several principles as recommended. A lowered postoperative hemoglobin was mainly caused by bleeding, but this was not the only indication for transfusion. The decision to transfuse a patient with packed red blood cells (PRBCs) was based on the patient's clinical condition rather than on a fixed hemoglobin threshold. Targeted transfusions were administrated according to the viscoelastic point-ofcare (POC) tests to improve the hemostatic capacity (35). In general, transfusions were administered based on the laboratory examination, patient's clinical situation and the doctor's experience.

The mechanisms of transfusion-related AKI remain unclear; however, several possible pathophysiological explanations have been proposed. The interrelated biochemical and morphological changes of erythrocytes can lead to depletion of adenosine triphosphate and 2,3-diphosphoglycerate, and small capillary obstruction during storage can influence tissue oxygen delivery. These changes may also lead to progressive hemolysis and accumulation of microparticles, cytokines, free hemoglobin, and iron in the supernatant, causing a proinflammatory state, exacerbating oxidative tissue stress, and contributing to organ tissue dysfunction transfused patients $(33,36,37)$.

To minimize the risk of AKI in these high-risk patients, the following measures should be considered: maintain perioperative hemodynamic stability, improve the quality of intraoperative hemostasis by reducing bleeding, avoid unnecessary perioperative blood transfusions; reduce the dose of contrast medium; avoid using nephrotoxic drugs.

This study had several limitations. First, this was a single-center retrospective study with small sample size. The cohort size in the AKI group was particularly small due to the limited number of patients treated with HCR and the low incidence of AKI in our center. Second, the differences between basic and intraoperative blood pressure, and the volume and composition of blood transfusions were not analyzed due to the small sample size, and these factors may play a role in the development of AKI.

\section{Conclusions}

In conclusion, one-stop HCR did not increase the incidence of postoperative AKI in our center. Advanced age ( $\geq 75$ years) and perioperative transfusions were independent risk factors for developing AKI after one-stop HCR. For elderly patients, more attention should be focused on perioperative kidney protection. The efficacy of a restrictive transfusion strategy at improving HCR outcomes by reducing AKI risk requires further verification. Future studies are warranted to identify and confirm the risk factors of AKI in patients undergoing HCR procedures.

\section{Acknowledgments}

Funding: This work was supported by Beijing Municipal Science \& Technology Commission Foundation (No. Z191100006619036).

\section{Footnote}

Reporting Checklist: The authors have completed the STROBE reporting checklist. Available at https://dx.doi. org/10.21037/apm-21-959 
Data Sharing Statement: Available at https://dx.doi. org/10.21037/apm-21-959

Conflicts of Interest: All authors have completed the ICMJE uniform disclosure form (available at https://dx.doi. org/10.21037/apm-21-959). The authors have no conflicts of interest to declare.

Ethical Statement: The authors are accountable for all aspects of the work in ensuring that questions related to the accuracy or integrity of any part of the work are appropriately investigated and resolved. The study was conducted in accordance with the Declaration of Helsinki (as revised in 2013). The study was approved by local research ethics board of Chaoyang Hospital (No.: 2021-D-5) and individual consent for this retrospective analysis was waived.

Open Access Statement: This is an Open Access article distributed in accordance with the Creative Commons Attribution-NonCommercial-NoDerivs 4.0 International License (CC BY-NC-ND 4.0), which permits the noncommercial replication and distribution of the article with the strict proviso that no changes or edits are made and the original work is properly cited (including links to both the formal publication through the relevant DOI and the license). See: https://creativecommons.org/licenses/by-nc-nd/4.0/.

\section{References}

1. Park SJ, Ahn JM, Kim YH, et al. Trial of everolimuseluting stents or bypass surgery for coronary disease. $\mathrm{N}$ Engl J Med 2015;372:1204-12.

2. Angelini GD, Wilde P, Salerno TA, et al. Integrated left small thoracotomy and angioplasty for multivessel coronary artery revascularisation. Lancet 1996;347:757-8.

3. Harskamp RE, Bonatti JO, Zhao DX, et al. Standardizing definitions for hybrid coronary revascularization. J Thorac Cardiovasc Surg 2014;147:556-60.

4. Rydén L, Ahnve S, Bell M, et al. Acute kidney injury after coronary artery bypass grafting and long-term risk of myocardial infarction and death. Int J Cardiol 2014;172:190-5.

5. McCullough PA. Contrast-induced acute kidney injury. J Am Coll Cardiol 2008;51:1419-28.

6. Cannon CP, Brindis RG, Chaitman BR, et al. 2013 ACCF/ AHA key data elements and definitions for measuring the clinical management and outcomes of patients with acute coronary syndromes and coronary artery disease: a report of the American College of Cardiology Foundation/ American Heart Association Task Force on clinical data standards (writing committee to develop acute coronary syndromes and coronary artery disease clinical data standards). J Am Coll Cardiol 2013;61:992-1025.

7. Khwaja A. KDIGO clinical practice guidelines for acute kidney injury. Nephron Clin Pract 2012;120:c179-84.

8. Gallagher S, Jones DA, Lovell MJ, et al. The impact of acute kidney injury on midterm outcomes after coronary artery bypass graft surgery: a matched propensity score analysis. J Thorac Cardiovasc Surg 2014;147:989-95.

9. Benedetto U, Luciani R, Goracci M, et al. Miniaturized cardiopulmonary bypass and acute kidney injury in coronary artery bypass graft surgery. Ann Thorac Surg 2009;88:529-35.

10. Kim K, Joung KW, Ji SM, et al. The effect of coronary angiography timing and use of cardiopulmonary bypass on acute kidney injury after coronary artery bypass graft surgery. J Thorac Cardiovasc Surg 2016;152:254-261.e3.

11. Machado MN, Miranda RC, Takakura IT, et al. Acute kidney injury after on-pump coronary artery bypass graft surgery. Arq Bras Cardiol 2009;93:247-52.

12. Shen W, Aguilar R, Montero AR, et al. Acute Kidney Injury and In-Hospital Mortality after Coronary Artery Bypass Graft versus Percutaneous Coronary Intervention: A Nationwide Study. Am J Nephrol 2017;45:217-25.

13. Lunyera J, Clare RM, Chiswell K, et al. Racial Differences in AKI Incidence Following Percutaneous Coronary Intervention. J Am Soc Nephrol 2021;32:654-62.

14. Marbach JA, Wells G, Santo PD, et al. Acute kidney injury after radial or femoral artery access in ST-segment elevation myocardial infarction: AKI-SAFARI. Am Heart J 2021;234:12-22.

15. Tsai TT, Patel UD, Chang TI, et al. Contemporary incidence, predictors, and outcomes of acute kidney injury in patients undergoing percutaneous coronary interventions: insights from the NCDR Cath-PCI registry. JACC Cardiovasc Interv 2014;7:1-9.

16. Kanchi M, Manjunath R, Massen J, et al. Neutrophil gelatinase-associated lipocalin as a biomarker for predicting acute kidney injury during off-pump coronary artery bypass grafting. Ann Card Anaesth 2017;20:297-302.

17. Maioli M, Toso A, Leoncini M, et al. Persistent renal damage after contrast-induced acute kidney injury: incidence, evolution, risk factors, and prognosis. Circulation 2012;125:3099-107.

18. Wang Y, Bellomo R. Cardiac surgery-associated acute kidney injury: risk factors, pathophysiology and treatment. 
Nat Rev Nephrol 2017;13:697-711.

19. Zhang F, Lu Z, Wang F. Advances in the pathogenesis and prevention of contrast-induced nephropathy. Life Sci 2020;259:118379.

20. Mehta RH, Honeycutt E, Patel UD, et al. Relationship of the time interval between cardiac catheterization and elective coronary artery bypass surgery with postprocedural acute kidney injury. Circulation 2011;124:S149-55.

21. Wintgen L, Dakkak AR, Shakaki MA, et al. Acute kidney injury following coronary artery bypass grafting and control angiography: a comprehensive analysis of 221 patients. Heart Vessels 2021;36:1-6.

22. Zhou S, Fang Z, Xiong H, et al. Effect of one-stop hybrid coronary revascularization on postoperative renal function and bleeding: a comparison study with off-pump coronary artery bypass grafting surgery. J Thorac Cardiovasc Surg 2014;147:1511-6.e1.

23. Zhao DX, Leacche M, Balaguer JM, et al. Routine intraoperative completion angiography after coronary artery bypass grafting and 1-stop hybrid revascularization results from a fully integrated hybrid catheterization laboratory/operating room. J Am Coll Cardiol 2009;53:232-41.

24. Chen SW, Chang CH, Chu Y, et al. Postoperative Renal Outcomes of On-Pump Beating-Heart versus Cardioplegic Arrest Coronary Artery Bypass Grafting. Acta Cardiol Sin 2017;33:542-50.

25. Weir MR, Aronson S, Avery EG, et al. Acute kidney injury following cardiac surgery: role of perioperative blood pressure control. Am J Nephrol 2011;33:438-52.

26. Coleman MD, Shaefi S, Sladen RN. Preventing acute kidney injury after cardiac surgery. Curr Opin Anaesthesiol 2011;24:70-6.

27. Yue Z, Yan-Meng G, Ji-Zhuang L. Prediction model for acute kidney injury after coronary artery bypass grafting: a retrospective study. Int Urol Nephrol 2019;51:1605-11.

28. Huang TM, Wu VC, Young GH, et al. Preoperative proteinuria predicts adverse renal outcomes after coronary

Cite this article as: Li D, Gao J, Guo Y, Jia Y, An X, Liu Y, Wang L, Su P. Risk factor analysis of acute kidney injury after one-stop hybrid coronary revascularization. Ann Palliat Med 2021;10(7):7398-7405. doi: 10.21037/apm-21-959 artery bypass grafting. J Am Soc Nephrol 2011;22:156-63.

29. Cohen E, Nardi Y, Krause I, et al. A longitudinal assessment of the natural rate of decline in renal function with age. J Nephrol 2014;27:635-41.

30. Chung SM, Lee DJ, Hand A, et al. Kidney function changes with aging in adults: comparison between crosssectional and longitudinal data analyses in renal function assessment. Biopharm Drug Dispos 2015;36:613-21.

31. Karrowni W, Vora AN, Dai D, et al. Blood Transfusion and the Risk of Acute Kidney Injury Among Patients With Acute Coronary Syndrome Undergoing Percutaneous Coronary Intervention. Circ Cardiovasc Interv 2016;9:e003279.

32. Rasmussen SR, Kandler K, Nielsen RV, et al. Association between transfusion of blood products and acute kidney injury following cardiac surgery. Acta Anaesthesiol Scand 2020;64:1397-404.

33. Karkouti K. Transfusion and risk of acute kidney injury in cardiac surgery. Br J Anaesth 2012;109 Suppl 1:i29-38.

34. Panoulas VF, Colombo A, Margonato A, et al. Hybrid coronary revascularization: promising, but yet to take off. J Am Coll Cardiol 2015;65:85-97.

35. Task Force on Patient Blood Management for Adult Cardiac Surgery of the European Association for CardioThoracic Surgery (EACTS) and the European Association of Cardiothoracic Anaesthesiology (EACTA); Boer C, Meesters MI, et al. 2017 EACTS/EACTA Guidelines on patient blood management for adult cardiac surgery. J Cardiothorac Vasc Anesth 2018;32:88-120.

36. Murphy GJ, Reeves BC, Rogers CA, et al. Increased mortality, postoperative morbidity, and cost after red blood cell transfusion in patients having cardiac surgery. Circulation 2007;116:2544-52.

37. Donadee C, Raat NJ, Kanias T, et al. Nitric oxide scavenging by red blood cell microparticles and cell-free hemoglobin as a mechanism for the red cell storage lesion. Circulation 2011;124:465-76. 